\title{
Human Retina Based Identification System Using Gabor Filters and GDA Technique
}

\author{
Shahad A. Sultan and M. F. Ghanim
}

\begin{abstract}
A biometric authentication system provides an automatic person authentication based on some characteristic features possessed by the individual. Among all other biometrics, human retina is a secure and reliable source of person recognition as it is unique, universal, lies at the back of the eyeball and hence it is unforgeable. The process of authentication mainly includes pre-processing, feature extraction and then features matching and classification. Also authentication systems are mainly appointed in verification and identification mode according to the specific application. In this paper, preprocessing and image enhancement stages involve several steps to highlight interesting features in retinal images. The feature extraction stage is accomplished using a bank of Gabor filter with number of orientations and scales. Generalized Discriminant Analysis (GDA) technique has been used to reduce the size of feature vectors and enhance the performance of proposed algorithm. Finally, classification is accomplished using k-nearest neighbor (KNN) classifier to determine the identity of the genuine user or reject the forged one as the proposed method operates in identification mode. The main contribution in this paper is using Generalized Discriminant Analysis (GDA) technique to address 'curse of dimensionality' problem. GDA is a novel method used in the area of retina recognition.
\end{abstract}

Index Terms - biometrics, human retina, Gabor filters, GDA, nonvascular, KNN.

\section{INTRODUCTION}

$\mathrm{M}$ any types of systems require reliable personal recognition methods to ascertain and determine the identity of individuals accessing various areas or services and data. These schemes ensure that the services and data are accessed only by authorized personnel. Systems that may use biometric recognition schemes include private areas of buildings, computer systems, government and military facilities and automated teller machines (ATM). Such areas are vulnerable to security violation and require high level of security to control such situations. Biometric Recognition is

Manuscript received March 10, 2020; revised September 1, 2020. Date of publication September 8, 2020. Date of current version September 8, 2020 This research was supported by Computer Engineering Department, College of Engineering, University of Mosul, Mosul, Iraq.

Authors are with the Computer Engineering Department, College of Engineering, Mosul, Iraq (e-mails: shahadali.sultan@gmail.com, mayada.faris@uomosul.edu.iq).

Digital Object Identifier (DOI): 10.24138/jcomss.v16i3.1031 defined as the automated authentication of individuals based on their physiological or behavioral characteristics. A high security level can be achieved by using this scheme as mentioned in references [1-11]. In this paper, the research concentrates on the use of retinal scans as means of authentication and human retina as the biometric. Proposed method improves the performance of retina based identification system by using Generalized Discriminant Analysis (GDA) technique as a solution for 'curse of dimensionality' problem which usually presents in the feature vector of biometrics. GDA is a novel method used in the area of retina recognition. It reduces the time complexity of classifier and increases the accuracy rate of the classification process. Since it only selects the most discriminant features from the feature vector. Improvement of the proposed method has been demonstrated by doing a comparison with other published research works. These works were in the area of retina recognition and used the same databases as shown later in Section V.

The retina is a layer of blood vessels that located at the back of human eye-ball. Since retina is not easily accessible, it is impossible to forge. Also it is not exposed to threats from the external environment, unlike other biometrics such as fingerprint, hand geometry, ear shape, face etc. The retina has a very distinct blood vessels form so that, every eye has its own totally unique pattern of this form. In addition, the eyes of identical twins are not identical in their retinal pattern. So, it is called 'Eye Print' $[5,12]$, these unique blood vessels form the foundation of the retinal recognition system. Blood vessels of retina remain stable over a person's lifetime unless the person subjects to a severe eye surgical operation or injury [1] [6-9] [13-16]. So, among all other biometrics, retina has the most fixed features for individual recognition. It is one of the most accurate and robust sources of authentication that can be used for highly sensitive areas.

Proposed method is designed to operate in identification mode. It uses Gabor filtering to extract nonvascular features from retinal image (to consume less computation time and build a real time identification system). During feature extraction phase; each retinal image is processed with Gabor filter bank. This bank consists of several two-dimensional (2D) Gabor filters with number of scales and orientations. 
Using Gabor filter bank, a robust three-dimensional (3D) feature vector is extracted from the retinal image. Since biometrics have a large number of features, dimensionality reduction techniques are needful to minimize the size of feature vectors. Generalized discriminant analysis (GDA) is used for this purpose. Finally, a simple k-nearest neighbor (KNN) classifier is used for classification. The overall proposed algorithm is shown in Fig. 1.

The rest of the paper is organized as the follows: Section II states a summary of relevant literature. Section III describes the anatomy of human retina. Section IV illustrates the proposed method. Section V discusses the experimental results. Section VI gives the conclusion of this research.

\section{RELEVANT LITERATURE}

With retinal authentication based on non-vascular features after preprocessing the features of the retinal image are extracted from retina without undergoing blood vessel segmentation, hence they are known as nonvascular features as these do not depend on blood vessels of retina only. It decreases the execution time of the system while preserving its good performance. Some of these methods are mentioned below:

Sabaghi et al. [17] have compensated the rotation of retinal image for a robust system. They have used FT (Fourier Transform) of the retinal image to extract features and called this feature vector as FSPF, and then they have used Euclidean

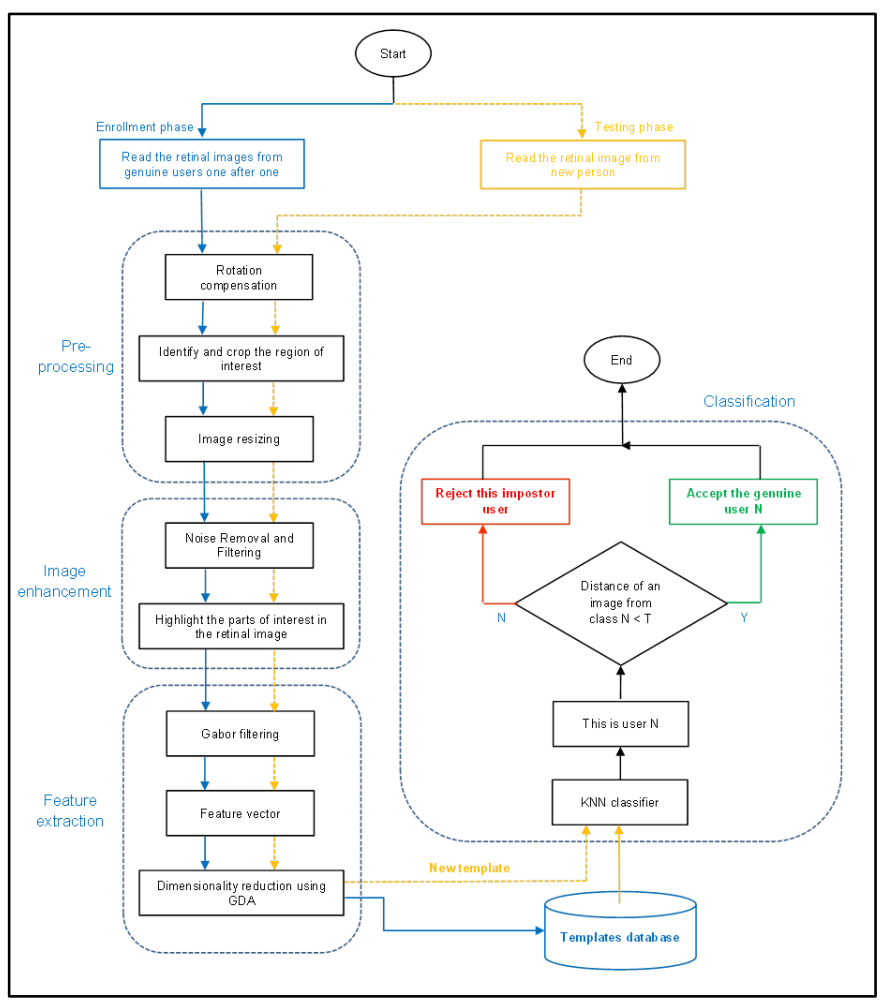

Fig. 1. Proposed algorithm for retinal based identification system

distance to perform matching process. Their method is robust to noise. In [18] Sabaghi et. al. have proposed a second method, which composes wavelet transform with Fourier transform to extract FSPF. Also they have used distance in matching phase. They have obtained accuracy rate of $95.4 \%$ with authentication system based on Fourier transform features and $97.2 \%$ with authentication system that based on wavelet transform features but they have obtained accuracy rate of $99.1 \%$ by using both techniques. Dehghani et. al. [2] have extracted features using Harris corner detector. They have used phase correlation method to determine the rotation of retinal image. Finally, they have evaluated a similarity function for matching process. Their method is more efficient because they have obtained accuracy rate of $100 \%$. Also their method consumes very low processing time. In [13] Ong et al. have proposed a graphical method for feature extraction where scale-invariant feature transform (SIFT) has been used. A subgraph matching algorithm has been used for matching process. They have generated DRIVERA (DRIVE for Retinal Authentication) database which contained 280 images that were created from 20 images of DRIVE database. They have used false acceptance rate (FAR) and false rejection rate (FRR) to evaluate the performance of their proposed method which are $0 \%$ and $3.169 \%$ respectively. They have showed that their system outperforms two of the high performance methods [4] and [19] when they evaluated their method on the same database. Waheed et al. [3] have proposed method that reads two images, to perform comparison at the same time, then they have evaluated Luminance and Contrast functions. They have incorporated luminance and contrast functions to get structure measurement. To evaluate a similarity value, an empirically optimized function is evaluated between two selected images. To evaluate their method, they have used only 34 subjects. Their method has got an identification rate of 92.5\%. In [8], Modarresi et al. have proposed retinal identification algorithm based on Shearlets transform feature extraction. They have used Radial Tchebichef Moments to eliminate the rotation effect from retinal image. They have also extracted the region of interest from retinal images in order to acquire similar parts from images that are belonging to the same person. In this method, Mahalanobis distance is used to estimate the degree of similarity between biometric patterns. Experimental results of this method show an equal error rate (EER) of 0.0024. In [20], B.M.S.Rani and A.Jhansi Rani have proposed biometric retinal security system for user authentication in smartphones. They have segmented blood vessels from retinal images and then measured the angle of bifurcation points in these vessels and the width of them. RNN (Recursive Neural Network) is used for matching process. Their method shows good performance and decreases the error rates.

\section{RETINAL ANATOMY}

Human retina has three main components: OD, macula and blood vessels as shown in fig 2-a. OD is brighter than other portions and is mainly circular in shape with a diameter of about $3 \mathrm{mms}$. It is also the entree and the exit point where nerves enter and quit the retina to and from the brain.

Macula or the "yellow spot" is the part of the retina that is most sensitive to light where it is responsible for our sharp central sight. It is located near the center of the retina about 2 


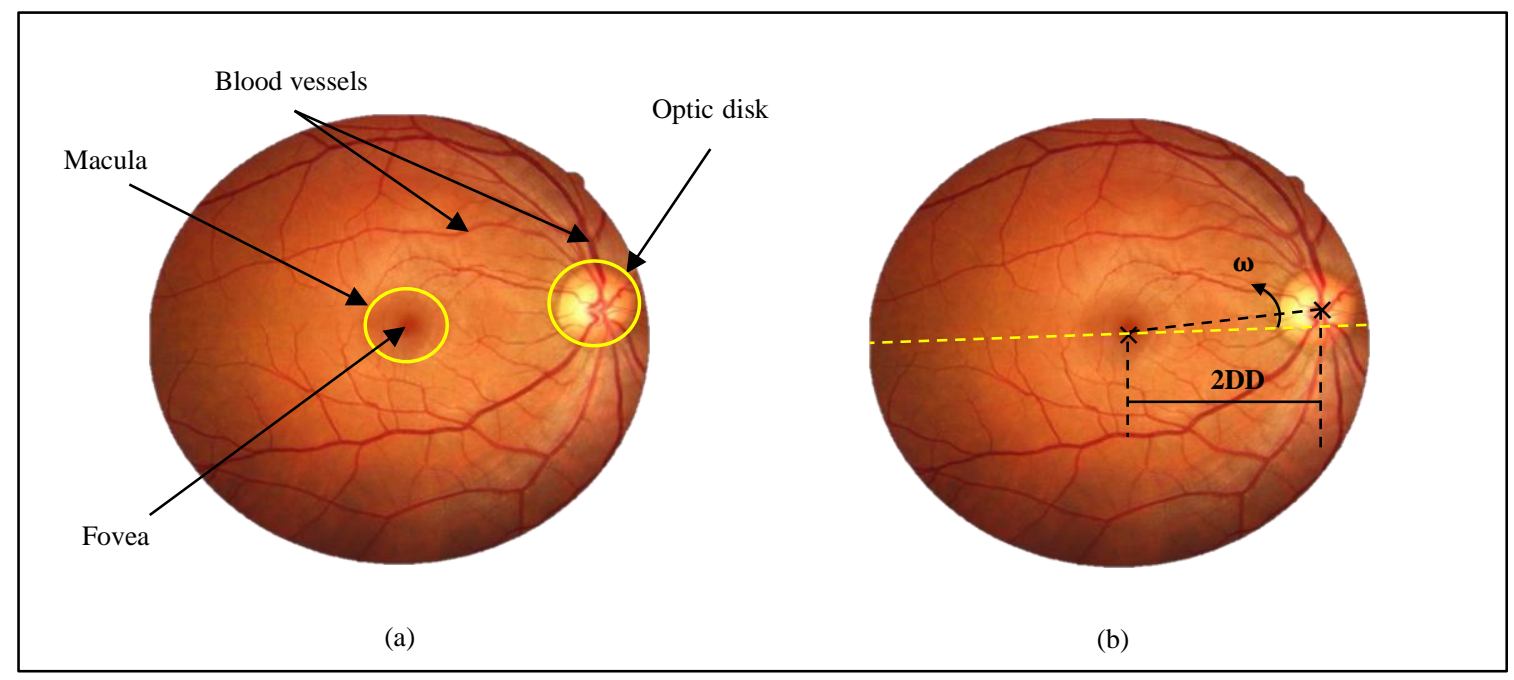

Fig. 2. Retinal Anatomy (a) Retinal main components (b) Position of macula and mean angle of fovea and center of the OD with the horizon

OD diameter (2DD) temporal to the OD. The darkest part of the macula called fovea, which is a very small region, forms the center of it. In the typical retinal image, the mean angle $(\omega)$ between fovea and center of the OD with the horizon is about $-5.6^{\circ}, \pm 3.3^{\circ}$ as shown in fig. 2-2-b. Blood vessels are patterns that have tree shape, branch from OD and continue on the surface of the retina. The mean diameter of the vessels is about $250 \mu \mathrm{m}[9][17][18]$.

\section{RESEARCH METHOD}

Any biometric authentication system has three main phases: pre-processing, feature extraction, and feature matching. The proposed method deals with these phases as shown in Fig. 1 which represents the complete algorithm of this research.

\section{A. Pre-processing}

This stage prepares the retinal image to correctly extract features later. The main problem related to the identification system based on human retina is that the retinal image can be affected by anatomic movement of eyeball or user head in front of the fundus camera during the acquisition process. This problem causes three different situations: scaling, rotation and translation in the retinal image. These three situations produce different images coming from the same person as shown in Fig. 3. So, to build a robust identification system it should be invariant to these three situations. Hence, pre-processing phase in the proposed method composes the following steps:

\section{Rotation compensation}

During this step, rotation angle of the retinal image is determined and then compensated to generate a rotation invariant template later in the feature extraction process. In the proposed method rotation angle has been estimated by localizing the center of the OD and the center of the macula and then determining the angle of the line that connects these two points (center of the OD and center of the macula) with the horizon. The measured angle represents $\omega+\beta$ and can be

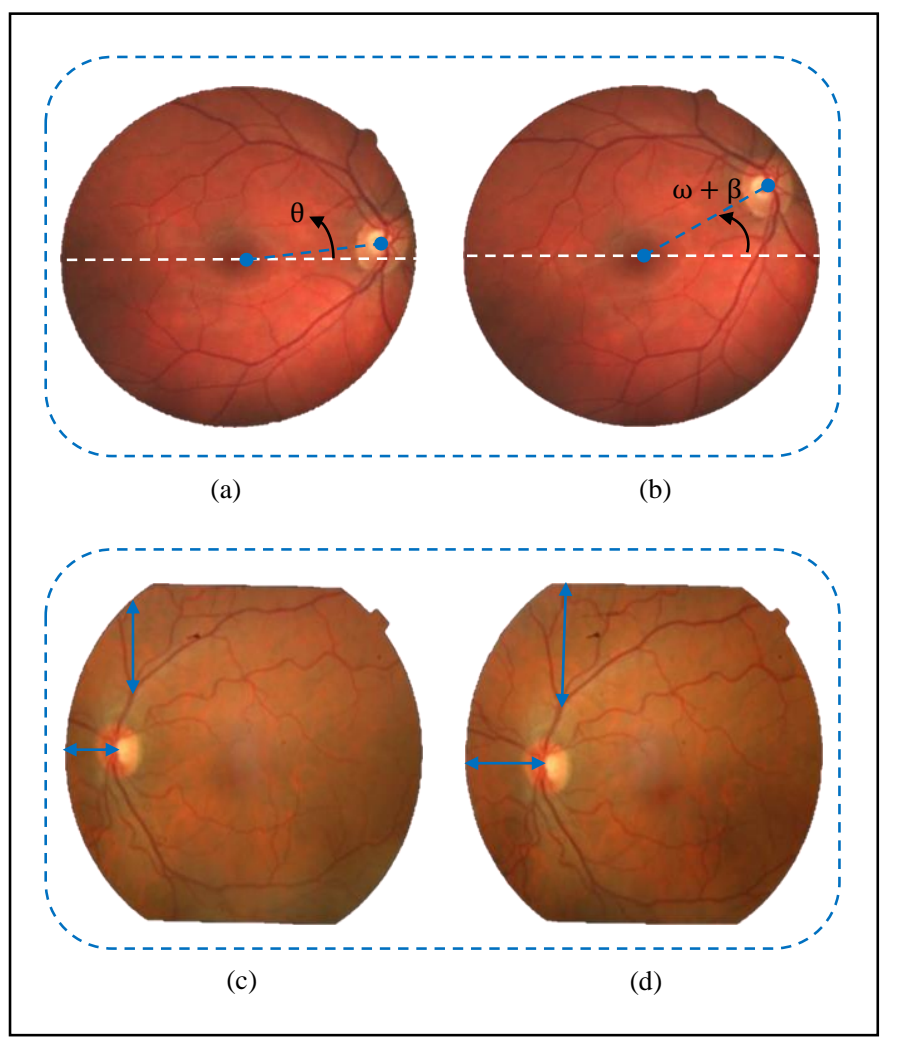

Fig. 3. Retinal image problems a) Retinal image without rotation b) Retinal image with rotation c) Retinal image before translation (d) Retinal image after translation

compensated by applying rotation in opposite direction on the input image. Compensation angle can be equal to $\omega$ or $\omega+\beta$. Whether rotation is compensated with $\omega$ or $\omega+\beta$, at the last all input images will be rotated to the same point. Rotation compensation process is illustrated in Fig. 4.

In the proposed method, template matching algorithm is used to localize the OD and determine the center point of it. Source image in the proposed method is the retinal image and template is the image of optic disc as shown in Fig 5.a and 5.c 


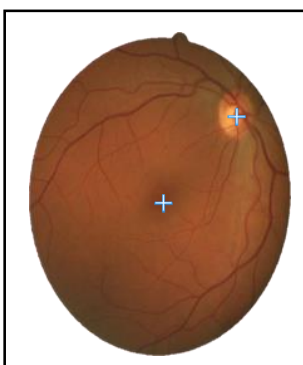

(a)

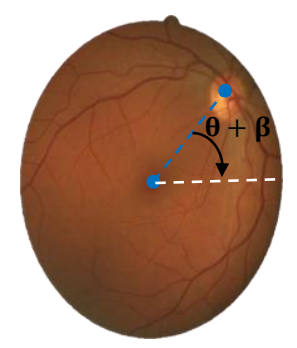

(b)

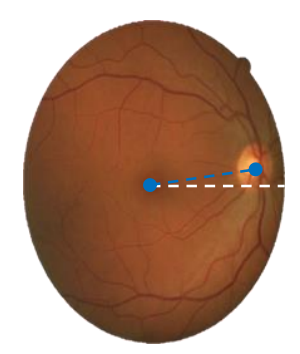

(c)
Fig. 4. Rotation compensation process (a) Determining the center of the optic disc and the center of the macula (b) Estimating the rotation angle (c) retinal image after compensating the rotation angle.

respectively.

In the proposed method, area-based matching approach is used and matching score is calculated using Normalized Cross Correlation (NCC). NCC is a matching score of two images with different sizes (source and template). Cross correlation process is analogous to convolution of two signals and NCC is a modern version of the cross correlation. It is invariant to changes in image intensities or noise level. It has a fast implementation which makes it widely used for template matching issues within real-time applications. Equation 1 illustrates the mathematical form of the NCC method [19, 20]:

$$
N C C=\frac{\sum_{x, y}\left[f(x, y)-\bar{f}_{u, v}\right][t(x-u, y-v)-\bar{t}]}{\left\{\sum_{x, y}\left[f(x, y)-\bar{f}_{u, v}\right]^{2} \sum_{x, y}[t(x-u, y-v)-\bar{t}]^{2}\right\}^{0.5}}
$$

$f(x, y)$ represents the source image; $\bar{f}$ is the mean value of source image intensities within the sliding window; $t$ represents the template image; $\bar{t}$ is the mean of template image intensities; $x, y$ and $u, v$ represents pixel coordinates [20].

In the proposed method, template matching algorithm is implemented using green channel of the retinal image and properly picked template image. This channel is used because it shows the pre-eminent contrast between blood vessels in retinal image and the retina itself. Template image is picked as a rectangular region around the OD. The result of implementing algorithm is illustrated in Fig 5. It determines a point that represents almost the center of the optical disc
(COD).

After localizing the OD, it is the time of localizing macula center point. Scientifically, fovea located at the Center of Mass (CoM) of retinal image. To find CoM of retinal image, it should be gone through pre-processing steps to remove the noise from this image and enhance its contrast thereby get more accurate results. These steps are as the following:

1) Each retinal image is resized and saved in JPEG format

2) Since the noise is only arised in the intensity component of the RGB images, this component is separated from the image. Separation is accomplished by converting the retinal image from RGB colour space to the HSV colour space. Then, the only value channel (V) is used in the following step.

3) Morphological structuring element processing is used to remove background noise from the $\mathrm{V}$ channel. Where, a flat structuring element is generated with disk shape and radius of 3 pixels. Open morphological operation eliminates, using this structuring element, snowflakes that have radius less than 3 pixels.

4) $\mathrm{V}$ component is filtered using the adaptive median filter to remove the noise from over this entire channel.

5) The contrast of $\mathrm{V}$ component is then enhanced using Contrast-limited adaptive histogram equalization (CLAHE) function.

6) The enhanced $\mathrm{V}$ channel is recombined with the $\mathrm{H}$ and $\mathrm{S}$ channels and then turned back to the RGB colour space.

7) RGB colour space image is converted to Lab colour space image. Lab is a wide colour space and more similar to the human eye system. Lab colour space provides a more convenient representation of colours compared to the RGB colour space which is designed specifically for display purposes. By trying several hypotheses on Lab colour space image and inspect its channels. Concatenation of ( $\mathrm{L}$ and $\mathrm{b}$ ) channels gives better results than using ( $\mathrm{L}$ with a) channels and even than using the entire Lab colour space.

After implementing pre-processing steps on retinal image, the result is shown in Fig 6, where Fig 6.c and 6.d represents retinal images after implementing pre-processing steps on images in Fig 6.a and 6.b respectively. At this point, calculating the CoM point is still affected by the remaining

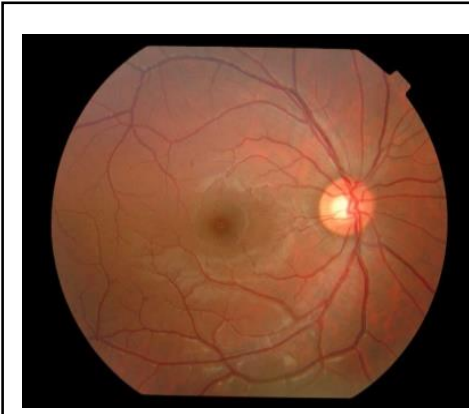

(a)

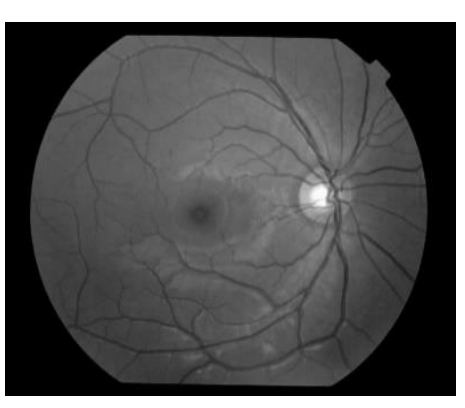

(b)

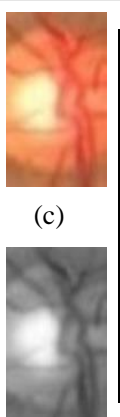

(d)

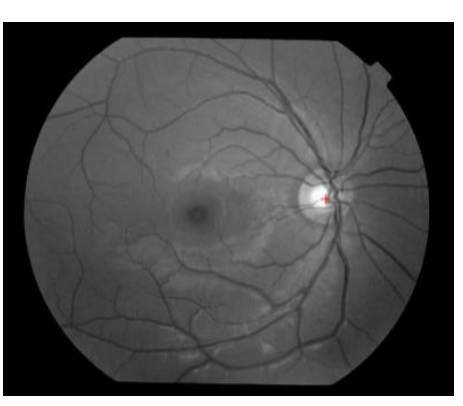

(e)

Fig. 5. Localization of optic disc using template matching algorithm (a) Retinal image from RIDB database (b) Green channel from retinal image (c) Template image (d) Green channel from template image (e)Result of localization. 


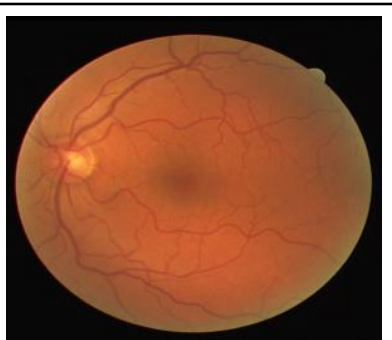

(a)

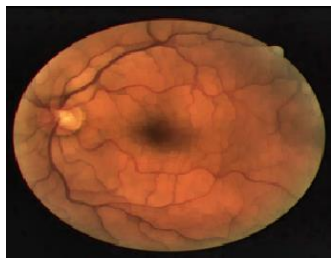

(c)

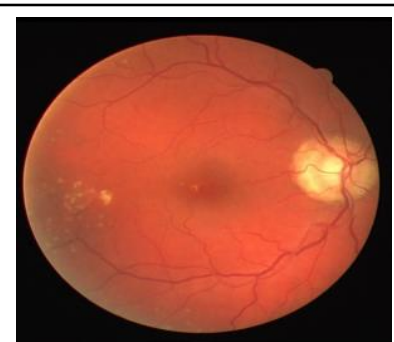

(b)

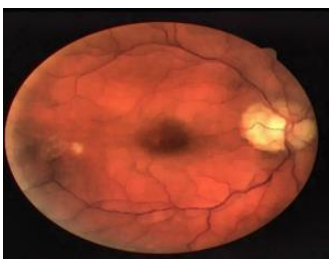

(d)
Fig. 6. Pre-processing results of fovea localization (a, b) Retinal images before pre-processing operation (c, d) Retinal images after preprocessing implementation.

noise and intensity inhomogeneity of the retinal image. To solve this problem, spatial information of retinal image is considered along with the intensity information. This gives extremely more accurate results than calculating the CoM point without considering spatial information of the retinal image (as doing in reference [18]). The enhancement of this modification is illustrated in fig. 7. The CoM is calculated using the following equations $[21]$ to get $(\bar{x}, \bar{y})$ coordinates of the center point:

$$
\bar{x}=\frac{\sum_{y=1}^{m} \sum_{x=1}^{n} x . f(x, y)}{\sum_{y=1}^{m} \sum_{x=1}^{n} f(x, y)}
$$

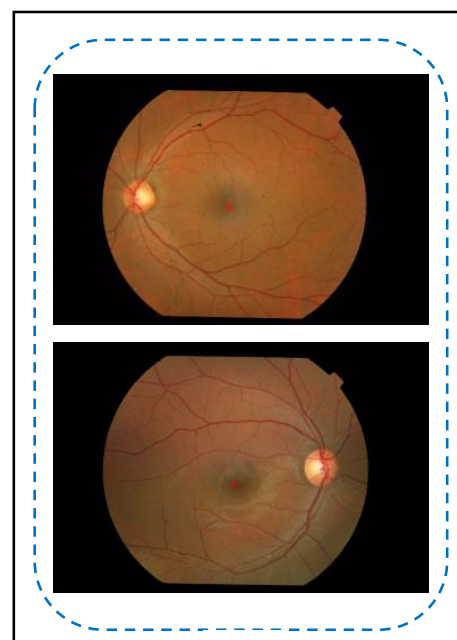

(a)

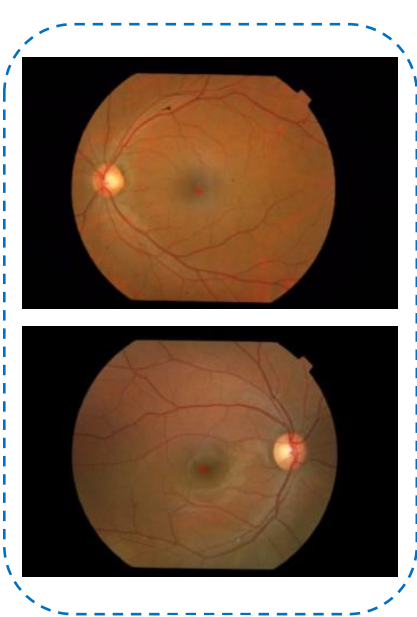

(b)
Fig. 7. Localization of macula center point on some retinal images (a) Using CoM based method without considering spatial information of the retinal image (c) Using CoM based method with considering spatial information of the retinal image.

$$
\bar{y}=\frac{\sum_{x=1}^{n} \sum_{y=1}^{m} y \cdot f(x, y)}{\sum_{y=1}^{m} \sum_{x=1}^{n} f(x, y)}
$$

At this point, the center of optic disc and the center of macula are located. The rotation angle of the line that connects these two points can be calculated using the following equation:

$$
\text { rotation_angle }=\tan ^{-1}\left[\frac{\left(y_{2}-y_{1}\right)}{\left(x_{2}-x_{1}\right)}\right]
$$

where $\left(x_{1}, y_{1}\right),\left(x_{2}, y_{2}\right)$ represent the center of macula and the center of optic disc respectively; rotation_angle is the angle of undesired rotation of the input retinal image.

The undesired rotation can be compensated by rotating the retinal image in opposite direction with the same angle.

\section{Cropping the region of interest}

Initially, blood vessels around OD have discriminative property more than those farther from OD so they are most important for authentication purposes. Blood vessels pattern around OD distributes with less randomness. As blood vessels go away from this region, they become thinner and more random in their distribution [9]. Also; after compensating undesired rotation of the retinal image there is a need to solve the translation problem as well. Translation in retinal image causes some parts from one image may not appear in another and vice versa (as shown in Fig 3.c and 3.d) which gives inaccurate results. So, region of interest (ROI) must be cropped from the retinal image to eliminate translation and achieve robust identification accuracy. This as well decreases the size of retinal image (without changing its resolution) and as a result reduces the processing time without affecting the accuracy rate.

To crop the ROI at first, template matching algorithm is used to localize the COD point (location of COD changed after rotation compensation process). The radius of the OD is determined to be 100 pixels; this value is considered as an average over the radius of optic disc in 25 retinal images from the used database. To crop a circular ROI with radius of 400 pixels from the retinal image, the center point of this region is

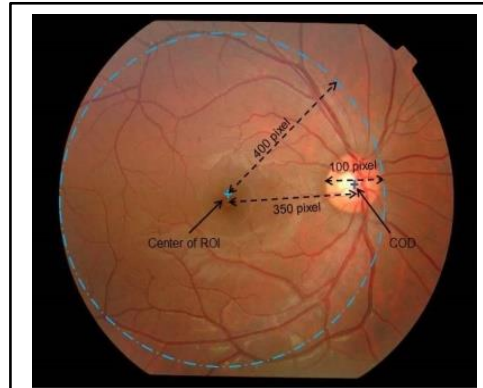

(a) (b)

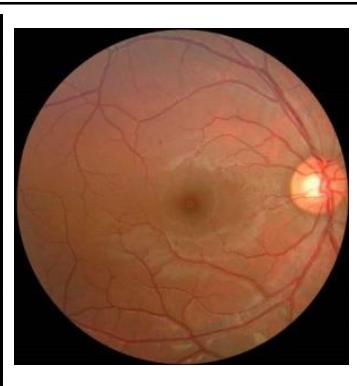

Fig. 8. Cropping the ROI (a) Determination of the ROI (b) The desired result after cropping the ROI 
determined at 350 pixels distance from the COD point to obtain a circular ROI as shown in Fig. 8. The resulting image contains mutual points of different images of the same individual; this meaning is illustrated in Fig. 9 which shows execution results of the proposed method (Fig. 9 (e \& j)). By cropping the ROI, accuracy rate of the proposed method improved from $97.05 \%$ to $100 \%$, which is considered a good improvement.

\section{Image resizing}

Image resizing represents the last step in the pre-processing phase. It is used to minimize the size of retinal image to enable processing of digital image to be accomplished with less computational time.

\section{B. Image enhancement}

This phase highlights the parts of interest from retinal image. It involves smoothing the intensity of different parts of the retinal image, sharpening, and removing the background noise that may be caused during the image acquisition process. Image enhancement makes the features of retinal image more visible and prepares it for features extraction phase.

\section{Morphological structuring}

Morphological structuring in the proposed algorithm is used to remove the background noise and some unwanted parts (features) from the retinal image. During this step, a flat structuring element is generated with disk shape and radius of 3 pixels. Morphological processing (open Morphological operation) uses this structuring element to eliminate snowflakes that have radius of less than 3 pixels. This eliminates thin bumps and small points that may be interfered with the major blood vessels in the retinal image.

\section{Image Sharpening}

This step performs process of de-blurring and increase the focus of the retinal image. This in turn highlights blood vessels and improves the contrast of the image.

\section{Histogram Equalization}

This process enhances the retinal image using its histogram. In the proposed algorithm, adaptive histogram equalization is used. Unlike histogram equalization, it processes small parts in the image in lieu of the entire image. It enhances each region in the retinal image separately then, the neighboring regions are combined. Using this process the contrast of the homogeneous parts can be restricted to obviate magnifying the noise that may be appeared in the retinal image.

\section{Noise Removal and Filtering}

Retinal image is liable to a wide range of noise. In retinal image, noise may be caused during the image acquisition process, during the transmission of image from acquisition hardware or as a result of how the image being transmitted. Linear filtering process is used to remove the noise from retinal image. It involves averaging filter and Gaussian filter. Averaging filter is used to remove grain noise and Gaussian filter which is a type of low pass filters used to remove the Speckle Noise that generally present in digital image. Image enhancement steps are illustrated briefly in Fig 10.

After implementing pre-processing and image enhancement steps, retinal image becomes as shown in Fig. 11.

\section{Feature extraction}

By implementing pre-processing and image enhancement processes on the retinal image, it becomes prepared for the feature extraction phase. During this phase, Gabor filter is used to extract non-vascular features from the retinal image. 2D Gabor filter is a Gaussian kernel function modulated by a complex sinusoidal wave. Gabor filters have two special merits which are not shared with other filters or methods of feature extraction. These merits are: frequencies and orientations depiction of Gabor filters are similar to those of

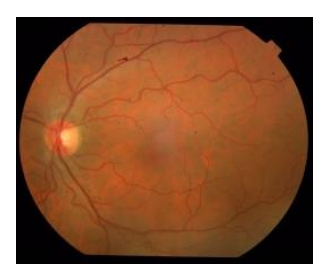

(a)

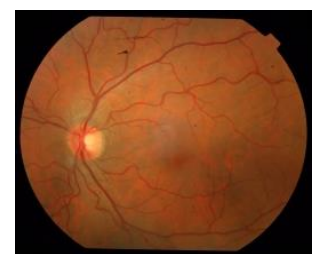

(f)

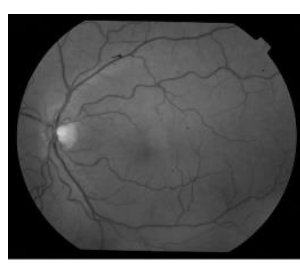

(b)

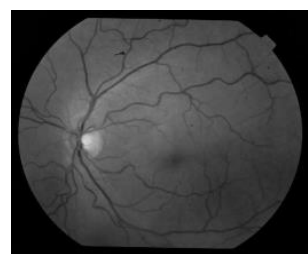

(g)

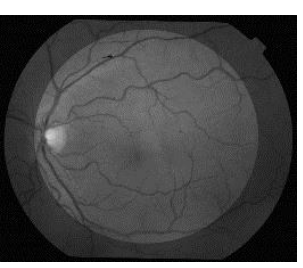

(c)

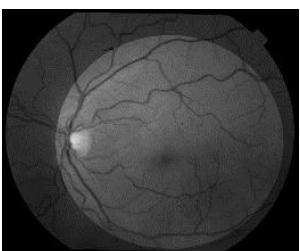

(h)

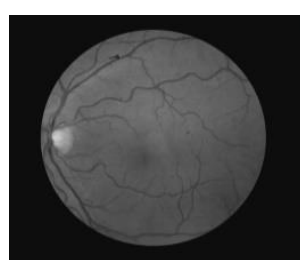

(d)

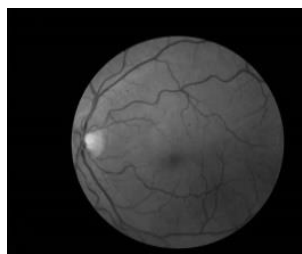

(i)

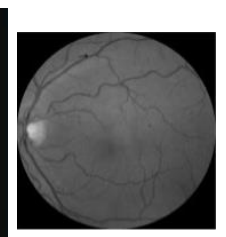

(e)

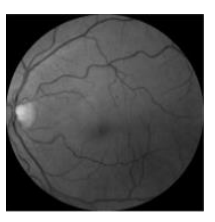

(j)

Fig. 9. Cropping the region of interest. (a) and (f) Retinal images from the same person. (b) and (g) Green channel from retinal images. (c) and (h) Determination of the ROI. (d) and (i) Final desired result. (e) and (j) Execution result after cropping the ROI using proposed method. 


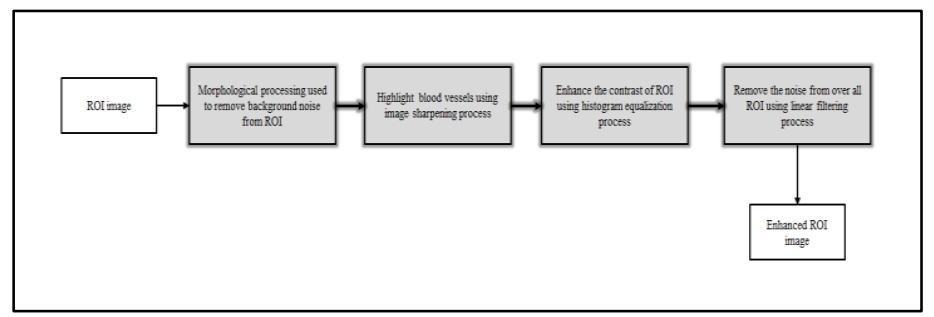

Fig. 10. Image enhancement process

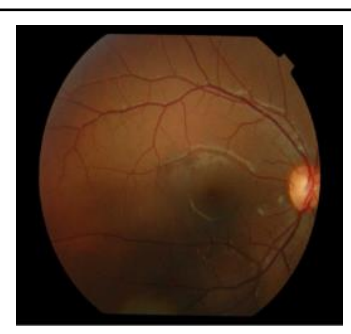

(a)

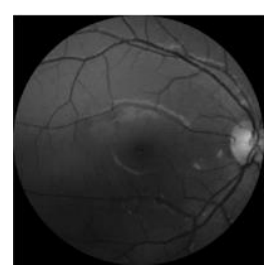

(b)

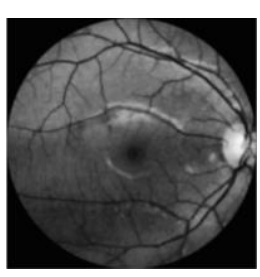

(c)
Fig. 11. Effect of preprocessing and image enhancement on retinal image (a) Retinal image from RIDB database (b) Pre-processing effect (c) Image enhancement effect

mammals' optical system. So, they are widely used to model the attitude of the primary visual cortex of mammals' optical system. The second reason of depending Gabor filters is their optimal space-frequency resolution [22]. Gabor filters have the ability to hold responses from texture at different scales and directions; this feature can be exploited to extract scale invariant features vector from the image [23]. Also, they are stable to change in photometric sensations like noise in the image and illumination effect. It is found that Gabor filters are particularly suitable for texture representation and pattern recognition [14], [22]. So, they are used in the proposed algorithm to extract features from retinal image. 2D Gabor filter is defined in the following equations [23].

$$
\begin{aligned}
G(x, y ; \lambda, \phi, \psi, \sigma, \gamma & =e^{\left(-\frac{x^{\prime 2}+\gamma^{2} y^{\prime 2}}{2 \sigma^{2}}\right)} e^{j\left(2 \pi \frac{x^{\prime}}{\lambda}+\psi\right)} \\
x^{\prime} & =x \cos \phi+y \sin \phi \\
y^{\prime} & =-x \sin \phi+y \cos \phi
\end{aligned}
$$

where, $x, y$ represent spatial coordinates of the $2 \mathrm{D}$ Gabor filter frequency; $\lambda$ represents the wavelength (inverse of spatial frequency) of the sinusoidal wave; $\phi$ represents the orientation of the parallel strips of Gabor function; $\psi$ represents the phase offset of the sinusoidal wave; $\sigma$ represents the standard deviation of the Gaussian envelope; $\gamma$ represents the spatial aspect ratio.

In the proposed algorithm, Gabor filter bank is composed of forty bandpass filters (in 8 directions and 5 scales) as shown in Fig. 12-a. Gabor filters with different orientations are used to extract features from retinal blood vessels which are presented at different directions. Different scales are used to eliminate scaling problem in retinal images.

This bank is applied to each input retinal image by convolving that image with each filter from the bank as shown in Fig 12-b. The results of the convolution process are then concatenated into a robust $3 \mathrm{D}$ feature vector. Feature extraction process is illustrated by using the following expressions:

$$
\begin{gathered}
v(x, y, z) \equiv V_{\phi(z), Q(z)}(I(x, y)) \\
V_{\phi(z), Q(z)}(I(x, y))=I(x, y) \otimes G_{\phi(z), Q(z)}(x, y) \\
Q(z)=f(z) \\
f(z) \in\left\{f_{\max }, \frac{f_{\max }}{\sqrt{2}}, \frac{f_{\max }}{(\sqrt{2})^{2}}, \frac{f_{\max }}{(\sqrt{2})^{3}}, \frac{f_{\max }}{\left(\sqrt{2}^{4}\right.}, \frac{5 \pi}{\left(\sqrt{2}^{5}\right.}\right\} \\
\phi(z) \in\left\{0, \frac{\pi}{8}, \frac{\pi}{4}, \frac{3 \pi}{8}, \frac{\pi}{2}, \frac{5 \pi}{8}, \frac{3 \pi}{4}, \frac{7 \pi}{8}\right\}
\end{gathered}
$$

where, $v(x, y, z)$ represents $3 \mathrm{D}$ feature vector; $I$ represents the

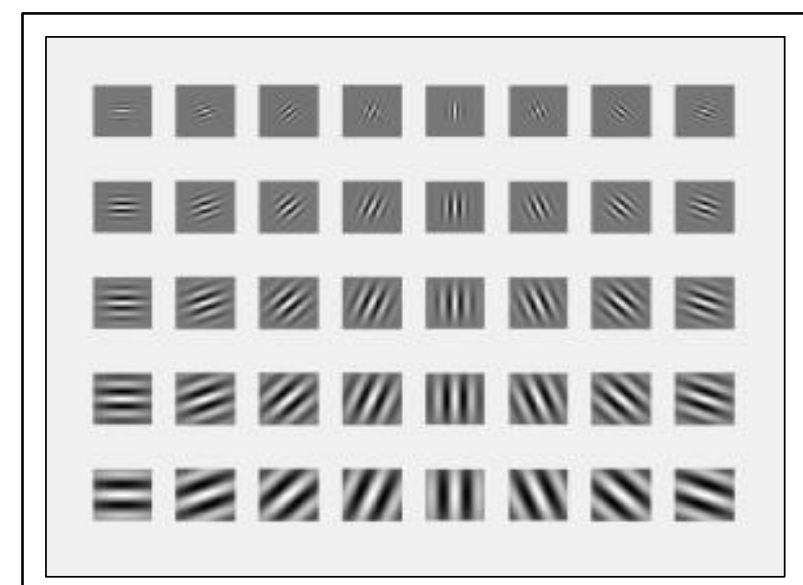

(a)

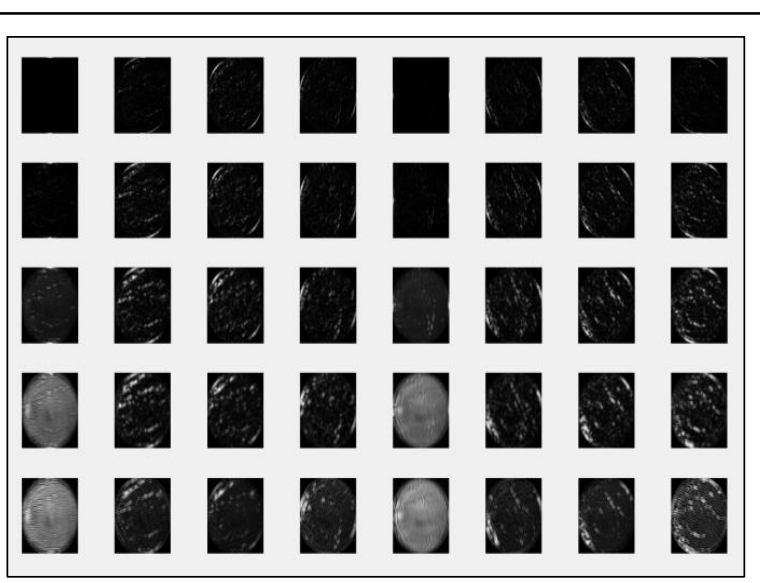

(b)

Fig. 12. Convolving retinal image with a bank of Gabor filters (a) Forty Gabor filters (in 8 directions and 5 scales) (b) Resultant images from Gabor filters 
input retinal image; $\mathrm{x}, \mathrm{y}$ represent pixel coordinates, $\mathrm{x} \in[1, \mathrm{n}]$; $\mathrm{y} \in[1, \mathrm{~m}] ; f(z)$ represent frequencies of scaled versions from Gabor function. Q (z) represent scales of Gabor filters. $G_{\theta(z), s(z)}(x, y)$ represents Gabor filter bank. $\phi(\mathrm{z})$ Represent orientations of Gabor filters.

The size of the resultant feature vector is related to the size of the retinal image, and the number of filters used in the Gabor filter bank. The size of the retinal image after image pre-processing and image enhancement phases becomes $120 \mathrm{x}$ 120 pixels. By convolving this image with forty filters, the size of feature vector becomes $(120 * 120 * 40=576000)$. Neighboring pixels in an image have high degree of correlation, so there is a need to reduce these redundant pixels and minimize the size of the feature vector. This can be accomplished by downsampling each of the resultant images from bank filters by a factor of 6 . After the downsampling process, the size of the feature vector becomes $(576000 /(6 * 6)$ $=16000)$.

As illustrated above, the size of the feature vector is large. It is very important to optimize the size of feature vector to guarantee response within deadline and generate a real time

\section{$F V_{i k}, F V_{j k} \quad$ Represent $i^{\text {th }}$ and $j^{\text {th }}$ feature vectors} respectively, each with length $M$.

identification system. Also, there is an essential need to eliminate the overlap among classes in a given dataset which greatly affects the accuracy rate of the designed system. One of the dimensionality reduction techniques can be used for this purpose.

\section{Dimensionality reduction of retina feature vector}

As shown in section 3.3, feature vector has a major length and contains ineffective information (those belong to the background of retinal image); this increases the computational time and reduces the accuracy rate of classification process. This problem is called 'curse of dimensionality' and so, there is a real need to remove the redundant features from the high dimensional feature vector and reduce the size of it.

Dimensionality reduction techniques project features of high dimensional onto a new generated axis. The most commonly used techniques, in the field of biometrics based authentication, are: Principle Component Analysis (PCA), and linear discriminant analysis (LDA). Such techniques are considered as classical dimensionality reduction techniques and each of them has its own drawbacks. PCA technique investigates in the data for directions that have greatest variance and then project the features into it to produce lower dimensional features vectors. PCA technique suffers from a number of problems which make it not the best solution for "curse of dimensionality" problem in the biometrics field. These problems are [23]: Linearity, Information packing transform, and it does not care about classes of a given dataset. LDA increases between class scatter and minimizes within class scatter which makes it shows a better classification performance than PCA technique but it also suffers from two essential problems: the linearity problem and the Small Sample Size (SSS) problem. These problems make it impossible to be implemented in the proposed system [24].

In this research, the main contribution is using Generalized Discriminant Analysis (GDA) technique to address 'curse of dimensionality' problem. GDA is a novel method used in the area of retina recognition. It is produced for non-linear classification and multi-class dataset. GDA is a kernel version of LDA where the original data space may not accept a linear separation. It is the more general case and used to eliminate any shortcomings of both the PCA and LDA techniques. With the GDA space the most valuable information is preserved which makes show high classification efficiency and reduces the training time of the used classifier [21][22][25][26]. Due to above reasons GDA is adopted, as a dimensionality reduction technique, in the proposed method.

\section{Classification process}

$\mathrm{K}-\mathrm{NN}$ classifier is used in the proposed system for the classification purpose. Euclidean distance (ED), given by the equation (10), is determined to measure the similarity score in $\mathrm{K}-\mathrm{NN}$ algorithm. K value is selected experimentally to be 4 .

$$
E D=\sqrt[2]{\sum_{k=1}^{M}\left(F V_{i k}-F V_{j k}\right)^{2}}
$$

\section{EXPERIMENTAL RESULTS AND DISCUSSION}

All experiments in this research work were conducted in the same environment which is composed of: Windows 10 Pro operating system, Intel (R) Core (TM) CPU @ $1.8 \mathrm{GHz}, 8 \mathrm{~GB}$ RAM, and Matlab (R2019b). Then, the system was tested using the following two databases:

1) Retinal Identification DataBase (RIDB) which was designed by Joddat Fatima, Adeel M. Syed, and M. Usman Akram during their research work in reference [5]. RIDB database contains 100 images of resolution $1504 \times 1000$ (collected from 20 different individuals with 5 images per individual).

2) Digital Retinal Images for Vessel Extraction (DRIVE) database. DRIVE database were acquired in the Netherlands from a diabetic retinopathy checking program. The checking people consisted of 400 diabetic persons between 25-90 years old. Forty images of resolution $768 \times 584$ pixels have been randomly opted from them.

To check the system in terms of rotation invariance, DRIVE database has been rotated. Rotation angles applied to retinal images are: $\pm 10^{\circ}, \pm 15^{\circ}, \pm 20^{\circ}, \pm 25^{\circ}, \pm 30^{\circ}, \pm 35^{\circ}$. After these rotation processes, number of images becomes 500. Fig. 13 shows some different images from the used databases.

Performance of the system has been evaluated using the following performance criteria:

- False Acceptance Rate (FAR): is the ratio of persons which are incorrectly accepted. Feature vectors of these individuals are matched to templates of non-matching users in the database. It can be expressed as: 


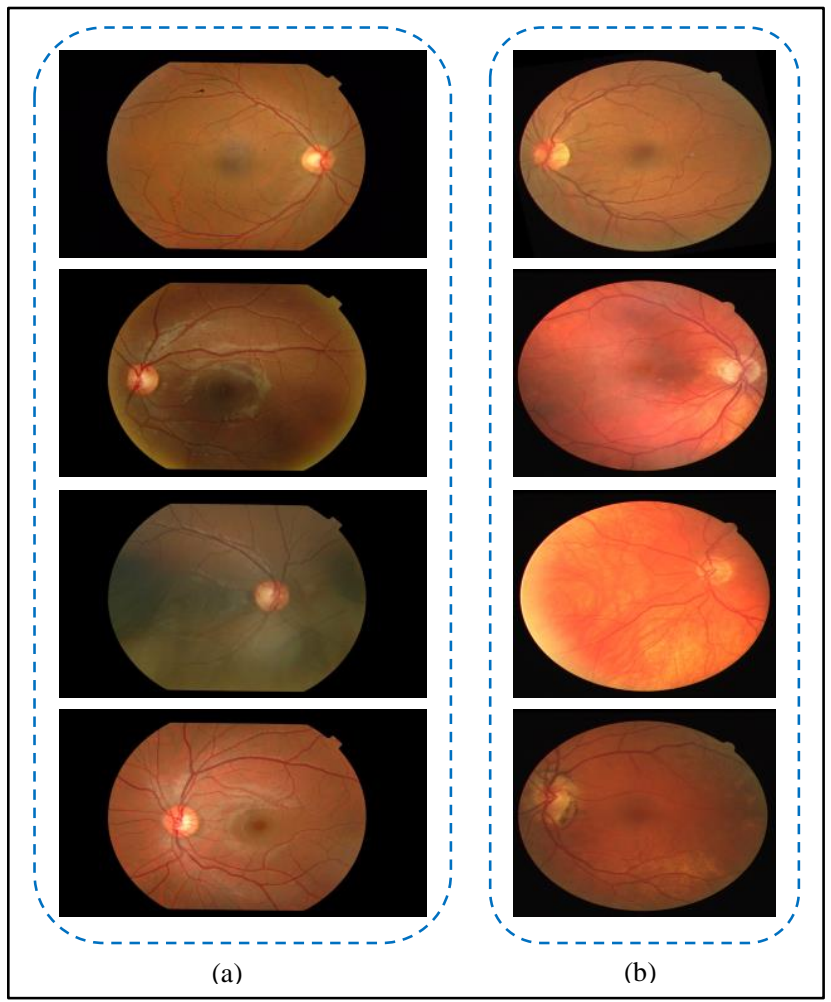

Fig. 13. Samples from used databases (a) Samples from RIDB database (b) Samples from DRIVE database.

$$
F A R=\frac{\text { number of accepted imposters }}{\text { number of imposter comparisons }} \times 100 \%
$$

- $\quad$ False Rejection Rate (FRR): is the ratio of persons which are incorrectly rejected. Feature vector of these individuals are not matched to their samples which already exist in the system database. It can be expressed as:

$$
F A R=\frac{\text { number of rejected genuine individuals }}{\text { number of genuine comparisons }} \times 100 \%
$$

- Receiver Operating Characteristic (ROC): is the curve that represents the relation between FAR and FRR. It represents a function of threshold value and abstracts the performance of the biometric authentication system. The tradeoff between FAR and FRR can easily be achieved using this curve.

- Equal Error Rate (EER): is the ratio at which both FAR and FRR have equal values. EER can easily be found out from the Receiver Operating Characteristic (ROC) curve. The most accurate system has the lowest EER value. EER can be computed at the point (on the ROC curve) where:

$$
\operatorname{FAR}(\mathrm{t})=\operatorname{FRR}(\mathrm{t})
$$

But practically, similarity score distributions are not continuous and the crossover point may not exist within these distributions. In this case ERR can be calculated as:

$$
E E R=\left\{\begin{array}{cc}
\frac{F A R\left(t_{1}\right)+F R R\left(t_{1}\right)}{2} & \text { if } F A R\left(t_{1}\right)-F R R\left(t_{1}\right) \leq F R R\left(t_{2}\right)-F A R\left(t_{2}\right) \\
\frac{F A R\left(t_{2}\right)+F R R\left(t_{2}\right)}{2} & \text { otherwise }
\end{array}\right.
$$

$$
\begin{aligned}
& t_{1}=\max _{t \in s}\{t \mid F R R(t) \leq F A R(t)\} \\
& t_{2}=\min _{t \in s}\{t \mid F R R(t) \geq F A R(t)\}
\end{aligned}
$$

$s$ is the set of threshold values used in the score distribution.

- Accuracy: is the rate of individuals that are correctly classified, accuracy of a given system is calculated as:

$\operatorname{Accuracy}(\%)=\left[100-\left(\frac{F A R(\%)+F R R(\%)}{2}\right)\right]$

So, accuracy of the system improves if FAR, FRR decreases.

In biometric systems, there is a tradeoff between FAR and FRR values. Values of these rates depend on the selected threshold value. If threshold value is brought down, FRR will increase (since the similarity score in the proposed system is the distance between two feature vectors) and FAR will decrease. When FAR and FRR for a given biometric system are computed against different threshold values (score distributions), the desired Operation Point (OP) of it can be easily selected $[5,6,27]$.

Fig. 14.a illustrates FAR and FRR values against threshold for the proposed identification system using RIDB database. This figure shows that the proposed system is not sensitive to threshold values in the range between 24 and 34. In this region, $F A R=F R R=E R R=$ zero, which represents the ideal performance for high security level application. Hence, the OP of the proposed system was determined to be here by making the threshold value of the classification process in the range of [24 to 34]. Fig. 14.b shows the ROC curve of the proposed system using RIDB database, it shows a good separation distance between the genuine and imposter class. The performance of the proposed identification system using DRIVE database is shown in fig. 14 (c and d). EER for both databases (RIDB and DRIVE) is zero. This value indicates the

\begin{tabular}{|c|c|c|c|c|c|c|}
\hline Method & Databases & $\begin{array}{c}\text { Number of } \\
\text { subjects }\end{array}$ & $\begin{array}{c}\begin{array}{c}\text { Number of } \\
\text { images }\end{array} \\
\end{array}$ & Accuracy $(\%)$ & PC specifications & $\begin{array}{l}\text { Computational } \\
\text { time (sec) }\end{array}$ \\
\hline \multirow{2}{*}{ Proposed method } & RIDB & 20 & 100 & 100 & \multirow{2}{*}{$\begin{array}{l}1.8 \mathrm{GHz} \mathrm{CPU} \\
8 \mathrm{~GB} \text { memory }\end{array}$} & 0.22 \\
\hline & DRIVE & 40 & 500 & 100 & & 1.2 \\
\hline $\begin{array}{l}\text { M. Modarresi et al. } \\
{[8]}\end{array}$ & DRIVE & 40 & 500 & EER $=0.0011$ & $\begin{array}{l}4.2 \mathrm{GHz} \mathrm{CPU} \\
8 \mathrm{~GB} \text { memory }\end{array}$ & 0.92 \\
\hline J. Fatima et al. [5] & RIDB & 20 & 100 & 97 & - & - \\
\hline E. P. Ong et al [13] & DRIVE & 20 & 280 & 98.42 & - & - \\
\hline P. Panchal et al. [7] & RIDB & - & 55 & 92.72 & - & - \\
\hline $\begin{array}{l}\text { B.M.S. Rani and } \\
\text { A.J. Rani [28] }\end{array}$ & DRIVE & - & - & 94 & $\begin{array}{c}\text { Device with the } \\
\text { Android OS }\end{array}$ & - \\
\hline $\begin{array}{c}\text { Zahra Waheed et al. } \\
\qquad 3]\end{array}$ & RIDB & 20 & 100 & 92.5 & - & - \\
\hline $\begin{array}{c}\text { T.S. Sasikala, K. S. } \\
\text { Sankar [29] }\end{array}$ & DRIVE & 40 & - & 83.8 & - & 16.4 \\
\hline
\end{tabular}
excellent performance of the proposed system. Finally, table 1 states comparison among the proposed method and other state

TABLE I

COMPARISON AMONG RESULTS OF PROPOSED METHOD AND OTHER IDENTIFICATION METHODS 


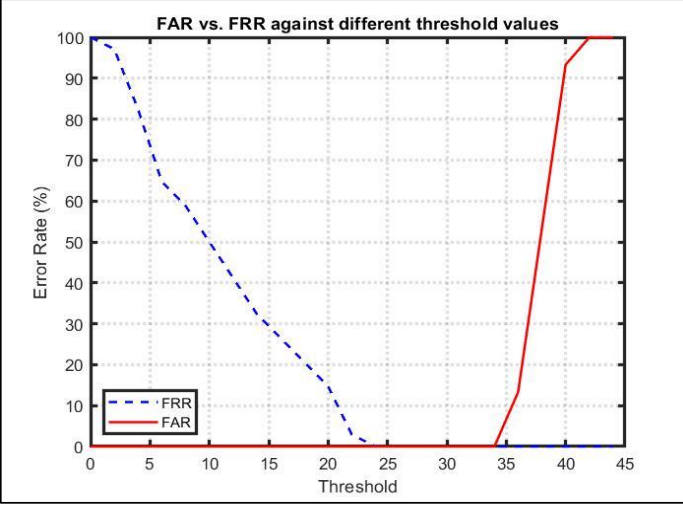

(a)

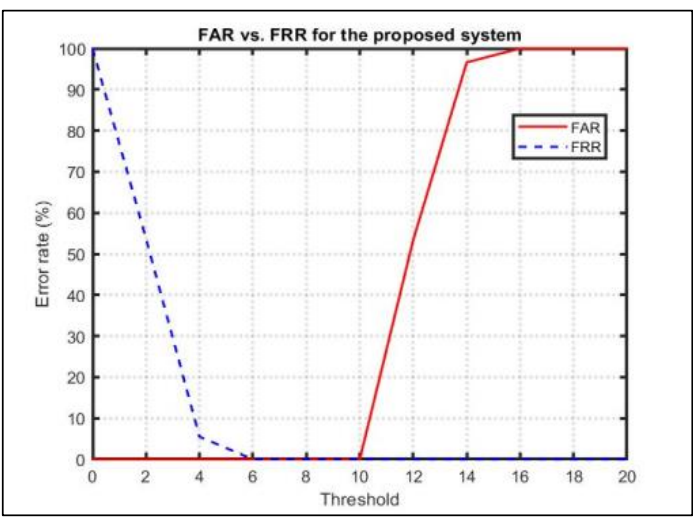

(c)

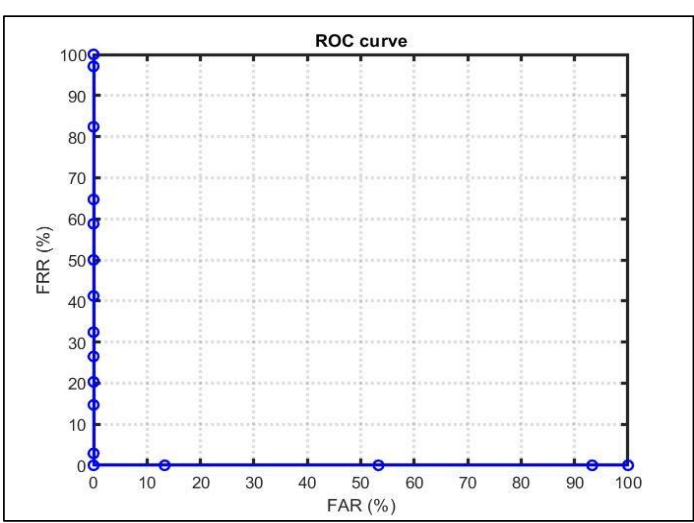

(b)

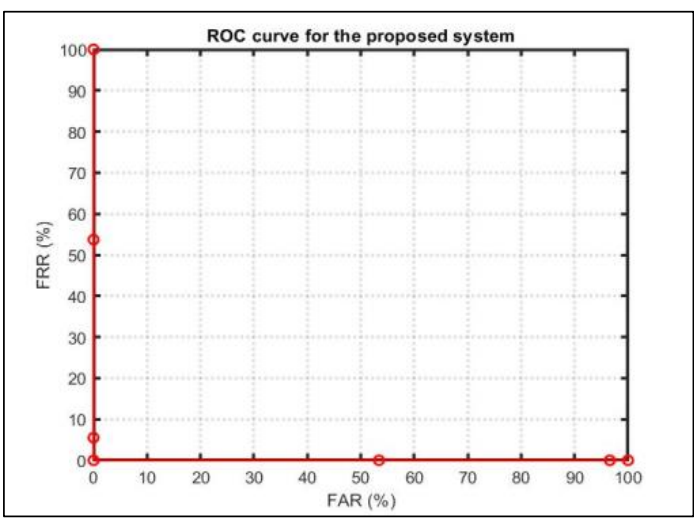

(d)

Fig. 14. Performance of the proposed system (a) FAR and FRR as functions of threshold values using RIDB database (b) ROC curve for the RIDB database (c) FAR and FRR as functions of threshold values using DRIVE database (d) ROC curve for the DRIVE database.

of the art published research works. To achieve a fair comparison, these works used the same databases presented in this research. From this table it can be seen that the proposed method outperforms other methods in terms of some criteria like accuracy and time consuming.

\section{CONCLUSION}

In This research a new algorithm is proposed for retinal identification system. A bank of Gabor filter with different scales and directions is used for feature extraction process and then GDA technique is used to diminish the length of feature vectors. This algorithm shows efficient performance as the accuracy of the proposed system is $100 \%$ for both RIDB and DRIVE databases. It outperforms many state of the art authentication systems.

\section{ACKNOWLEDGEMENT}

The authors are grateful to University of Mosul and Computer Engineering department for their support in carrying out this research.

\section{REFERENCES}

[1] Jarina B, S. R. Nirmala. Retina based Biometric Authentication System. International Journal of Advanced Research in Computer Science. DOI 10.26483/ijarcs.v9i1.5322. 2018; 9(1): 711-718.

[2] A. Dehghani, Z. Ghassabi, H. A. Moghddam, M. S. Moin. Human recognition based on retinal images and using new similarity function. EURASIP Journal on Image and Video Processing. 2013; 2013(1): 1-10.

[3] Z. Waheed, A. Waheed, M. U. Akram. A robust nonvascular retina recognition system using structural features of retinal image. 13th International Bhurban Conference on Applied Sciences and Technology (IBCAST). Bhurban. 2016: 101-105.

[4] H. Oinonen, H. Forsvik, P. Ruusuvuori, O. Yli-Harja, V. Voipio, H Huttunen. Identity verification based on vessel matching from fundus images. IEEE International Conference on Image Processing. Hong Kong. 2010: 4089-4092.

[5] J. Fatima, A. M. Syed, M. U. Akram. A secure personal identification system based on human retina. 2013 IEEE Symposium on Industrial Electronics and Applications (ISIEA). Kuching. DOI: 10.1109/ISIEA.2013.6738974. 2013: 90-95.

[6] Sheela S, V.R Udupi, Rahul D. Biometric Verification, Security Concerns and Related Issues. I.J. Information Technology and Computer Science. DOI: 10.5815/ijitcs.2016.04.06. 2016: 42-51.

[7] Parth P, Ronak B, Tejendra P. An Algorithm for Retinal Feature Extraction using Hybrid Approach. Procedia Computer Science. 7th International Conference on Communication, Computing and Virtualization. Gujarat. DOI: 10.1016/j.procs.2016.03.009. 2016: 61-68.

[8] Modarresi M, Oveisi IS, Janbozorgi M. Retinal Identification using Shearlets Feature Extraction. Journal of Austin Biometrics and Biostatistics. 2017; 4(1): 1-8. 
[9] Rostom Kachouri, Mohamed Akil, Yaroub Elloumi. Retinal image processing in Biometrics. In: Amine Nait-ali. Hidden Biometrics When Biometric Security Meets Biomedical Engineering.1. Singapore: Springer, Singapore. 2019: 1- 22.

[10] T.S. Sasikala , K. Siva Sankar. Unimodal Biometric Based Security Application by Exploiting Retina. International Journal of Engineering and Advanced Technology (IJEAT). 2018; 8(2S): 344-353.

[11] S.sravan Kumar, N.Anand Ratnesh. A Review on Different Biometric Techniques: Single and Combinational. IOSR Journal of Electronics and Communication Engineering (IOSR-JECE). DOI: 10.9790/28341104012530. 2016; 11(4): 25-30.

[12] C. K"ose, C. 'Iki, et al. A personal identification system using retinal vasculature in retinal fundus images. Expert Systems with Applications. DOI:10.1016/j.eswa.2011.04.141. 2011: 13670-13681.

[13] E. P. Ong, Y. Xu, D. W. K. Wong, J. Liu. Retina verification using a combined points and edges approach. Image Processing (ICIP). IEEE International Conference. Singapore. DOI: 10.1109/ICIP.2015.7351297. 2015: 2720-2724.

[14] Mohamed A, M. Hassaballah, Mohammed A. Identity Verification of Individuals Based on Retinal Features Using Gabor Filters and SVM. Journal of Signal and Information Processing. http://dx.doi.org/10.4236/jsip.2016.71007. 2016: 49-59.

[15] Shaydyuk, N. K., Cleland T. Biometric Identification via Retina Scanning With Liveness Detection Using Speckle Contrast Imaging. 2016 IEEE International Carnahan Conference on Security Technology (ICCST). Orlando. 2016: 1-5

[16] M. Suganya, K. Krishnakumari. A Novel Retina based Biometric Privacy using Visual Cryptography. IJCSNS International Journal of Computer Science and Network Security. 2016; 16(9): 76-80.

[17] Masoud Sabaghi, S. Reza Hadianamrei, Ali Zahedi, Maziyar Niyakan Lahiji. A New Partitioning Method in Frequency Analysis of the Retinal Images for Human Identification. Journal of Signal and Information Processing. DOI:10.4236/jsip.2011.24039. 2011; 2(4): 274-278.

[18] Masoud Sabaghi, S. Reza Hadianamrei, Mehdi Fattahi, Mohammad Reza Kouchaki, Ali Zahedi. Retinal Identification System Based on the Combination of Fourier and Wavelet Transform. Journal of Signal and Information Processing. http://dx.doi.org/10.4236/jsip.2012.31005. 2012; 3(1): 35-38

[19] A. P. Condurache, J. Kotzerke, A. Mertins. Robust retina-based person authentication using the sparse classifier. Signal Processing Conference (EUSIPCO). Bucharest. 2012: 1514-1518.

[20] B. M. S. Rani, A. J. Rani. Biometric Retinal Security System for user Identification and Authentication in Smartphones. International Journal of Pure and Applied Mathematics. 2018; 119(14): 187- 202.

[21] G.R. Prashantha, Chandrashekar M. Patil. An Approach for the Early Detection of Retinal Disorders and Performing Human Authentication. Proceedings of International Conference on Cognition. DOI: DOI 10.1007/978-981-10-5146-3 16. Singapore. 2017: 157-173.

[22] S. N. Kayte. Design and Development of Non-Proliferative Diabetic Retinopathy Detection Technique using Image Features Extraction Techniques. Master's thesis. Aurangabad: Dr. Babasaheb Ambedkar Marathwada University. Department of Computer Science and Information Technology; 2013.

[23] Nazanin Sadat Hashemi, Roya Babaei Aghdam, Atieh Sadat Bayat Ghiasi, and Parastoo Fatemi. Template Matching Advances and Applications in Image Analysis. American Scientific Research Journal for Engineering, Technology, and Sciences (ASRJETS). 2016; 26(3) 91-108.

[24] A. Grinshpan and J. Golabek. Locating Centers of Mass with Image Processing. Undergraduate Journal of Mathematical Modeling: One + Two. DOI: https://doi.org/10.5038/2326-3652.10.1.4906. 2019; 10(1): 124.

[25] Rached Belgacem, Hédi Trabelsi, Ines Malek, Imed Jabri. Applying a Set of Gabor Filter to 2D-Retinal Fundus Image to Detect the Optic Nerve Head (ONH). Annals of Medical and Health Sciences Research. 2018; 8: 48-58.

[26] Mohammad Haghighat, Saman Zonouz, Mohamed Abdel-Mottaleb. CloudID: Trustworthy cloud-based and cross-enterprise biometric identification. Expert Systems with Applications. http://dx.doi.org/10.1016/j.eswa.2015.06.025. 2015; 42(21): 7905-7916.

[27] A. Tharwat, Linear discriminant analysis: A detailed tutorial, AI Communications. DOI 10.3233/AIC-170729. 2017; 30(2): 169-190.

[28] D. J. Bora, A. K. Gupta, and F. A. Khan. Comparing the Performance of $\mathrm{L}^{*} \mathrm{~A} * \mathrm{~B} *$ and HSV Color Spaces with Respect to Color Image
Segmentation. International Journal of Emerging Technology and Advanced Engineering. 2015; 5(2): 192-203.

[29] Y. Zhang, D. Yeung. Semi-Supervised Generalized Discriminant Analysis. IEEE Transactions on Neural Networks. 30 Jun. 2011; 22(8): 1-11. DOI: 10.1109/TNN.2011.2156808.

[30] A. J. Mansfield, J. L. Wayman / Dave Rayner. Best Practices in Testing and Reporting Performance of Biometric. National Physical Laboratory. 14/02. 2002

[31] T.S. Sasikala, K. S. Sankar. Cascaded Biometric System Based on Fingerprint and Retina for User Identity Recognition. International Journal of Innovative Technology and Exploring Engineering (IJITEE). Apr. 2019; 8(6S4): 1357- 1363.

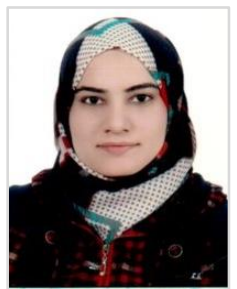

Shahad Ali Sultan is presently working as researcher to get master degree in computer engineering from Mosul University. She did her bachelor's degree in computer engineering/ University of Mosul, 2014. She presented "Implementation of Voice Equalizer using Tms320c6711" as final year project. Her area of interest is computer security.

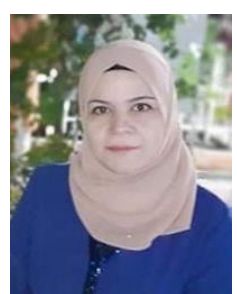

Mayada Faris Ghanim has experiences in wireless and mobile communications and computer security. Her educational attainments are $\mathrm{BSc}$ and $\mathrm{MSc}$ from Computer Engineering Department, College of Engineering at University of Mosul, Mosul, Iraq, her PhD. from Faculty of Electrical and Electronic Engineering at University Tun Hussein Onn Malaysia, Malaysia. 